\title{
Modelling and Simulation of UWB Radar System for Through the Wall Imaging and Doppler Detection
}

\author{
Ayesha Qamar" ${ }^{\# 1}$ Umar Faruq ${ }^{\# 2}$ \\ ${ }^{I}$ Student (M.Tech), Department of Electronics and Communication, \\ Quba college of Engineering and Technology, Nellore, A.P. INDIA. \\ ${ }^{2}$ HOD and Associate Professor, Department of Electronics and Communication, \\ Quba college of Engineering and Technology, Nellore, A.P. INDIA.
}

\begin{abstract}
In recent years, Through the Wall Imaging (TWI) has become a topic of research interest due to its promising applications in home-land security situations. In this paper, the basic concept of Stepped-Frequency Continuous Wave (SFCW) for ranging the targets behind the wall is described. Imaging of targets is implemented using the back-projection algorithm. This paper also brings out the received signal model for detection of stationary human being behind the wall using SFCW approach. The detection is based on estimation of heart beat and respiratory motion. Simulation results of human detection through Doppler processing and the 2-D imaging of targets are brought out for illustration. MATLAB software has been used for the development of signal processing modules.
\end{abstract}

Keywords - Stepped Frequency Continuous Wave, Through the wall imaging, Ultra wide band, Back projection, Through the wall radar, Doppler Processing, Field Programmable Gate array, MATLAB.

\section{INTRODUCTION}

For many years humans have dreamt of seeing through opaque objects such as wall. However, wavelengths of visible light allow transparent view through only very small kinds of materials. On the other hand, Ultra Wideband (UWB) electromagnetic technology with frequencies of few giga hertz has emerged as a potential candidate to be able to penetrate through almost all types of materials around us. Detection of humans behind the wall is of foremost interest for many applications. One such scenario could be in the hostage rescue situations, where detection and location of humans inside a room is very critical. The rescuers have a daunting task of unknown building layouts together with presence of armed persons in such alarming situations. Another use could be for disaster search and rescue operations such as people trapped under building debris during earthquake, explosion or fire.

In such situations a Through the Wall Radar (TWR) sensor is expected to detect moving, as well as stationary objects inside the building, classify targets, and possibly provide inputs on the dimensions/geometry of the building. Information gathered by the radar should be rendered in human readable form such as 1-D (down range only), 2-D (down-range vs. cross-range) with appreciable accuracy. The design should cater for low power transmission low probability of interception), packaged for compactness, lesser weight, and it should be battery powered. The detection of human beings through the wall is a complex problem and offers a formidable design challenge that necessitates a sensitive, wide-band width receiver to illuminate the target scene.

TWR designs have followed both time (impulse) and frequency domain (FMCW) waveform approaches. Impulse radar works in the time domain [1] and obtains high range resolution by transmitting a wide band impulse which means the pulse width will be in the order of few nano seconds. The disadvantage with this method is the requirement of high sampling rates and complex hardware design. The frequency domain approach such as Frequency Modulated Continuous Wave (FMCW) and SFCW works on generating the required bandwidth by means of modulating the transmitted wave.

The approach used in this paper uses SFCW radar, which synthesizes an impulse from a finite spectrum [2]. The main advantage of the stepped-frequency technique is that it is relatively easy with current technologies to efficiently sample SFCW signals with low speed analog-to-digital converters due to very low instantaneous bandwidth. Also, due to the transmission of long duration waveforms, a high average transmitted power is much easier to obtain than for short-pulse and impulse waveforms and it is also jammer resistant. The disadvantage of the SFCW approach had been the acquisition time. As SFCW system has to step through a number of frequencies for an acquisition of one A-Scan and for each Ascan an IFFT has to be calculated.

The SFCW radar scans the environment by transmitting the EM waves and tries to identify the targets behind the wall. The wave after transmitting through the antenna passes through the wall, strikes the target objects, gets reflected back, passing through the wall again and are received at the receiving antenna. By capturing these re-flections, we can image objects behind a wall. Building a device that can capture such reflections, however, is difficult because the signal power after traversing the wall twice (in and out of the room) is reduced by three to five orders of magnitude [3]. Even more challenging are the reflections from the wall itself (in stand-off mode of operation) or for that matter transmit to receive antenna mutual coupling, both of which result in much stronger signals in the receive chain than the reflections from objects inside the room ([4]-[5]). Reflections off the wall overwhelm the receiver's Analog to Digital Converter (ADC), preventing it from registering the minute variations due to reflections from objects behind the wall because of limited dynamic range. This behaviour is called the "Flash Effect" since it is analogous to how a mirror in front of a camera 
reflects the camera's flash and prevents it from capturing objects in the scene.

The detection of humans requires the processing of Doppler information from the SFCW wave. The working principle says that when a human body is exposed under the incidence of a Continuous Wave microwave source, the reflected signal is phase-modulated due to the (periodic) chest movements caused by respiration and heartbeat [6]. The de-modulated phase after the required processing gives information which contains information about movement due to heartbeat and respiration. This idea is extended to a SFCW system where we collect the return phase values over many sweeps. This collected phase values over sweeps gives the Doppler information.

The paper is organized as follows. Section II describes the SFCW Radar in and its specifications. In section III presents the mathematical modeling of human targets pertaining Doppler information. Back Projection algorithm is explained in section IV. Section V is devoted to results and Section VI for conclusion.

\section{SFCW RADAR}

Stepped frequency $\mathrm{CW}$ radar incorporates an RF source or a direct digital synthesis (DDS) to generate the waveform. The source is stepped between a start frequency, $f_{0}$ and a stop frequency, $f_{N-1}$, in equal, linear increments as shown in Fig. 1. It is important to note that for swept FM-CW radar, the source is swept from $f_{\min }$ to $f_{\max }$ with synthesized bandwidth $\mathrm{B}$ and linearly sampled. In either case, the radar is continuously transmitting. A return signal is formed by mixing the received signal with a portion of the transmitted one. This return signal is digitized at each step and stored. After each complete sweep of $\mathrm{N}_{\mathrm{f}}$ steps, an inverse Fourier transform is performed to convert the data from the frequency domain to the time domain. This is the process of creating the synthesized pulse.

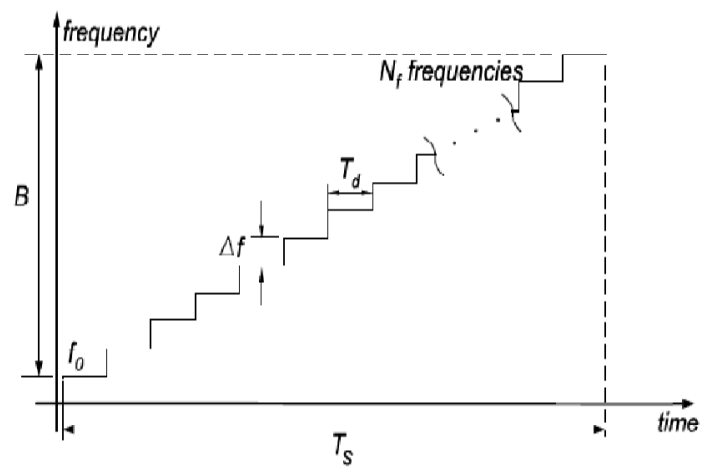

Fig. 1. Transmitted signal frequency profile.

In this paper a homodyne configuration of SFCW architecture is used. Range information is based on the timeof-flight principle, which is a phase path difference measurement. The portion of transmitted signal and received signal is mixed to generate a zero Intermediate Frequency (IF). The block diagram of the configuration is shown in Fig. 2.

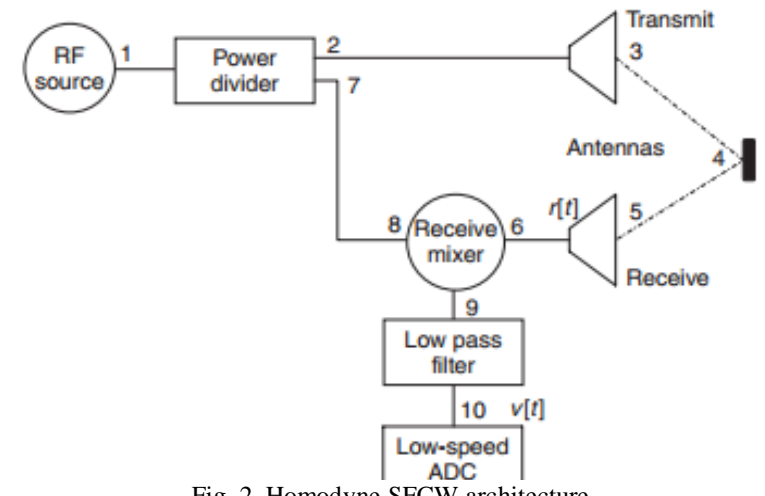

Fig. 2. Homodyne SFCW architecture

When the RF source is stepped in equal, linear increments of $\Delta f$ from $\left(f_{0}, f_{1}, \ldots f_{N-1}\right)$, the output voltages $(v[0], v[1], v[2]$,. .., $v[N-1])$ sampled at each step resemble a sampled sinusoid.

$$
v(n)=A \sum_{i=0}^{N-1} \delta(n-i) \cos \left(\varphi_{i} n\right)
$$

where $A$ and $\varphi_{i}$ are amplitude and phase indices corresponding to the complex reflectivity coefficient of a target at $i^{\text {th }}$ frequency, where $\varphi_{i}=2 \pi\left(f_{i}+n \Delta f\right) \tau_{0}, \tau_{0}$ is the delay corresponding to target range. The step size $\Delta f$ decides the maximum unambiguous range the radar can detect. The lower the step size the higher the unambiguous range and vice versa. The resolution which is the ability to distinguish the two closely spaced targets is determined by the bandwidth $(N \Delta f)$.

At each step, $n$, one sample is collected is from the ADC, $v[n]$. The data from all $N$ steps are then converted into the time domain pulse response equivalent with an Inverse Discrete Fourier Transform (IDFT). In case of multiple targets, the received signal can be modelled as a mixture of sine waves of different frequencies, as the rate of change of phase increases with range of the target. The phase of the received echo signal scattered from a target at range $R$ relative to transmitted sinusoid is, $\varphi=\frac{4 \pi F R}{c}$, where $\mathrm{R}$ is range to target, $\mathrm{F}$ is the transmitted frequency, $\mathrm{c}$ is the velocity of EM propagation. Evaluation of the first derivative of the above phase term provides insight into the value of phase information of the received signal.

An IFFT applied over these phase values gives the range to the target and can be expressed in terms of sinc function.

$$
v(t)=\frac{A}{2} B \frac{\sin (\pi B(t-\tau))}{\pi B(t-\tau)} \cos \left(2 \pi f_{c}(t-\tau)\right)
$$

Where $\mathrm{B}$ represents bandwidth of the transmitted signal, while $f_{c}=\frac{f_{\max }+f_{0}}{2}$ is the centre frequency of the waveform. The amplitude term is often referred to as the point spread function (PSF) of the radar waveform.

The main advantages of stepped frequency continuous wave radar is its high dynamic range and low noise floor as well as the ability to avoid certain frequencies when 
transmitting, thus making compliance with licensing requirements much easier.

\section{MICRO-DOPPLER PROCESSING OF SFCW RADAR}

Mechanical vibrations or rotations of structures on a target may introduce frequency modulation on the radar return signal. The modulation due to this vibration or rotation is referred as micro-Doppler (m-D) phenomenon. For example, human physical activity is a complex motion that is comprised of movements of individual body parts, including head, torso, leg, arm and foot. When human is walking, the movements of different components of human body generate a number of different frequency shifts in the radar returned signal, also called Doppler frequency shifts. The method proposed here analyses the phase modulation that a sinusoidal signal generated by means of a proper continuous wave transceiver, undergoes when reflected by the chest periodic movement associated with breathing as shown in Fig. 3.

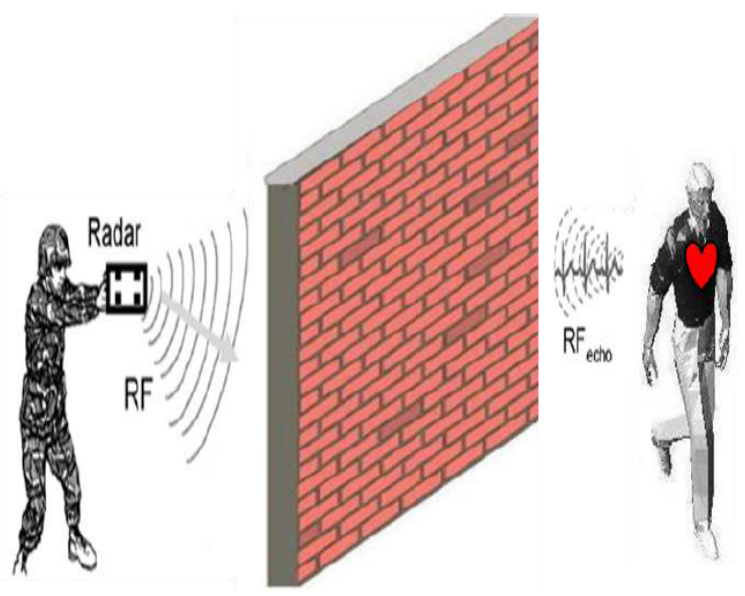

Fig. 3. Reference scenario for Micro-Doppler processing

The mathematical model of a Doppler processing for continuous wave radar is followed by the SFCW radar. Consider a UWB continuous radar that sends out a periodic signal with a frequency ' $f$ ' and the evolution versus time of a signal $\mathrm{T}$ generated by a $\mathrm{CW}$ system can be modelled as

$$
T(t)=A(t) \cos (2 \pi f t+\emptyset(t))
$$

where $A(t)$ is the amplitude of the signal, $f$ indicates the carrier frequency, and $\varnothing(\mathrm{t})$ is the phase noise of the oscillator. The continuous signal is sent out by stationary radar and is reflected by a target that is moving towards the radar at a constant velocity $v$. Let us suppose that the signal $\mathrm{T}$ in Eq.(3) is backscattered by a target placed at a distance equal to $\mathrm{d}_{0}$ from the $\mathrm{CW}$ system, and characterized by a local time varying displacement, referred to as $\mathrm{x}(\mathrm{t})$, related to chest movement. In this condition the total distance between the transmitting and receiving antenna of a $\mathrm{CW}$ system is equal to

$$
2 d(t)=2 d_{0}+2 \mathrm{x}(\mathrm{t})
$$

The chest movement $\mathrm{x}(\mathrm{t})$ can be expressed as contributions from respiration and heart rate. The received signal [6] can be approximated as

$$
R(t) \cong \cos \left(2 \pi f t-\frac{4 \pi \mathrm{d}_{0}}{\lambda}-\frac{4 \pi x(t)}{\lambda}+\varnothing\left(t-\frac{d_{0}}{c}\right)\right)
$$

where $c$ stands for the speed of the light, and $\lambda$ is the signal wavelength equal to c/f. In particular the received signal shows a time evolution similar to $\mathrm{T}(\mathrm{t})$, but a time delay due to two different contributions: the former is due to the nominal distance $d_{0}$ the latter is associated with the periodic motion of the target.

The setup for detecting the Doppler is similar to as explained for the SFCW system. The received signal is mixed with the local copy of the local oscillator signal, derived from the transmitted signal, proper information about the target periodic motion can be gained. A Quadrature demodulator can be implemented to decompose the signal into both I and Q components. If we consider only the $I$ component of the signal by multiplying Eq. (5) with $\cos (2 \pi \mathrm{ft})$ and low pass filtering we can express the resultant signal as

$$
B(t)=\cos \left(\theta+\frac{4 \pi x(t)}{\lambda}+\Delta \emptyset(t)\right)
$$

Where $\theta=\frac{4 \pi d_{0}}{\lambda}+\theta_{0}$ and $\Delta \emptyset(t)=\emptyset(t)-\emptyset\left(t-\frac{2 d_{0}}{c}\right)$ stand for the constant phase shift due to distance $d_{0}$ and the residual phase noise.

The phase signal can be obtained by

$$
\alpha=\theta+\frac{4 \pi x(t)}{\lambda}+\Delta \emptyset(t)
$$

Eq. (7) signifies the fact that the phase of the signal depends on the $\mathrm{x}(\mathrm{t})$, which is the combination of two signals related to heart beat and respiration. Therefore, without loss of generality $x(t)$ is assumed in the following to be a single tone signal, i.e. $x(t)=\sin (\omega t)$. Where $\square$ denotes the angular frequency of the chest movement.

The phase-modulated signal by substituting $x(t)=$ $\sin (\omega t)$ into Eq. (7) can be expressed as

$$
B(t)=\operatorname{Re}\left\{\exp ^{j\left(\frac{4 \pi m \sin (\omega t)}{\lambda}\right)} \exp ^{j(\theta+\Delta \emptyset(t))}\right\}
$$

The exponential term in Eq. (8) is expanded using Fourier series as

$$
\exp ^{j\left(\frac{4 \pi m \sin (\omega t)}{\lambda}\right)}=\sum_{n=-\infty}^{\infty} J_{n}\left(\frac{4 \pi m}{\lambda}\right) \exp ^{j n \omega t}
$$

Where $J_{n}(x)$ is the nth order Bessel function of the first kind, an interesting Fourier-series based representation of the phase modulated signal can be obtained by substituting Eq.(9) into Eq.(8) and is described as 


$$
B(t)=\sum_{n=-\infty}^{n=\infty} \operatorname{Jn}\left(\frac{4 \pi m}{\lambda}\right) \cos ((n \omega t+\theta+\Delta \emptyset(t))
$$

Based on Eq. (10) the phase modulated signal is decomposed into frequency components which is $\mathrm{n}$ times the basic frequency of the periodic movement and can be analysed in frequency domain. This concept was extended to step frequency radar by considering multiple sweeps with a periodic repetition rate T. The base band signal in Eq. (10) is periodic and can be sampled at a fast-sampling interval of $T s$. The sampled data at the $p$ th PRT is saved at the $\mathrm{p}^{\text {th }}$ column of two-dimensional matrix $s[l, p]$, where $l$ is the row number (i.e., range bin) while $p$ is the column number (i.e., slow-time index). Assuming the target movement/motion happens at range bin $l$, the sample time for the $l^{\text {th }}$ range bin and $p^{\text {th }}$ pulse is calculated as $t_{l, p}=p T+T_{0}+l T_{s}$, where $T_{0}$ is the initial time. Since $s[l, p]=z_{n}\left(t_{l, p}\right)$, the recorded signal sample will be

$$
\begin{gathered}
s[l, p]=\sum_{n=-\infty}^{n=\infty} J n\left(\frac{4 \pi m}{\lambda}\right) \cos \left(\left(n \omega\left(p T+T_{0}+l T_{s}\right)\right)+\theta\right. \\
+\Delta \emptyset(t))
\end{gathered}
$$

For $M$ receiving antennas, the data set will be extended to three-dimension shown as $s[l, p, m]$, where $m$ indicates that the data is captured by the $m$ th receiving antenna. Fast Fourier transform (FFT) is applied to the slow-time sampled signal, i.e., the row of $s[l, p]$, to extract the Doppler frequency shift. The column (i.e., range bin) $l$ is selected based on if it includes any target motion. This model is incorporated in the simulations with a human target and the results are illustrated in section $\mathrm{V}$.

\section{RADAR IMAGING}

SFCW radar introduced in earlier section only operates as a rangefinder where the information about the target is known. No further information about the exact location of a backscattering object is obtained. It is only known that it lies on a circle with the sensor as centre and the measured range as radius. The axis perpendicular to the antenna axis is called Down Range and the axis parallel to the antenna axis is called Cross Range. To accomplish the task of mapping, using a single UWB sensor is therefore not sufficient. Multiple sensors are necessary to get cross range information about the target. The angle of arrival from the different sensors needs to be integrated to find the final position of the target with respect to the sensor location. The most popular and effective techniques used in Radar imaging are the Back projection and Back propagation (Migration) Techniques [3]. Back projection technique has its origin in Synthetic Aperture Radar (SAR) and Back propagation techniques like Kirchhoff Migration \& Stolts (f-k) Migration [4] have their roots in Seismology. In this paper, Back projection algorithm is used to image targets behind the wall.

The data from each received signal consist of a set of Electric field values as function of the time travelled by the radar signals. The signal received at a given time can be from all pixel locations where the total flight time is equal to this specific time bin. The total flight time is the time to travel from the transmitting antenna to the pixel and then back to a receiver. The back projection techniques consists of recording the amplitude of each time bin on a spatial grid based on the total flight time, after that, all the recorded amplitudes from each channel are coherently added together on the spatial grid. By the phrase coherent summation it is meant that the signal obtained at each aperture position is time-shifted to match, or align, it to a particular pixel element in the image map. Following this, the responses across all aperture positions are combined.

To sum up, the position of an object can be estimated by using an antenna array in combination with the back projection algorithm. The distance from each antenna to the object is measured. The back projection method then sums up the range profiles of each antenna element. The exact echo waveform does not necessarily have to be known. The image plane is considered to be as $f(x, y)$ as shown in Fig.4. The $x-$ axis denotes direction parallel to parallel axis and $y$-axis denotes the direction perpendicular to antenna axis.

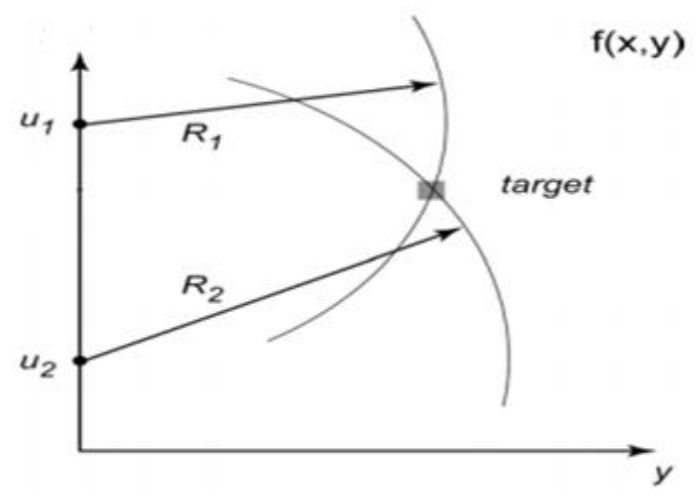

Fig. 4. Illustration of Back Projection algorithm

The back projection algorithm has been implemented as follows:

1. Divide the whole region into small surface areas or pixels. 2. For each pixel, calculate the total flight time from transmitter to pixel and pixel to a receiver.

3 . Record the corresponding received time bin amplitude for each pixel.

4. Repeat step 2 and step 3 for all receivers.

A main drawback of this algorithm is that each of these point responses smears the beam pattern of the image in the azimuth direction. The larger the distance of the target to the array centre, the worse the smearing distortion. To solve the problem of smearing distortion, the correlation of the receiver channels with a reference channel is proposed in literature. Preferably, the reference point response is measured away from the centre of the receiving array.

\section{RESULTS}

In this section the results of the SFCW processing with the operating parameters are discussed. A homodyne architecture based TWR receiver is assumed in the simulation. The sweep 
Bandwidth of the SFCW signalling is assumed to be $500 \mathrm{MHz}$ which yields a range resolution of $0.3 \mathrm{~m}$. The step frequency $\Delta f$ is considered to be $10 \mathrm{MHz}$ which corresponds to 50 steps for the entire bandwidth. Two sets of simulations were carried out using the above set of parameters. The Transmitter and receiver antennas are assumed to be collocated and they are placed at a distance of $7.5,8,8.5,9 \mathrm{~m}$ respectively along the $\mathrm{x}$ axis (Cross Range) and the two dimensional imaging will be presented. The red colour indicates the maximum intensity and indicates the position of the target, and the blue colour indicates the minimum intensity.

\section{A. Scenario 1:}

In the first scenario, the sensor array is located at $7.5 \mathrm{~m}, 8 \mathrm{~m}$, $8.5 \mathrm{~m}$ and $9 \mathrm{~m}$ respectively. Three objects were placed at a distance of $(8.5,2) \mathrm{m}$ corresponding to target1, $(7.5,5) \mathrm{m}$ corresponding to target 2 and $(9,7) \mathrm{m}$ corresponding to target 3 along the cross-range and down-range direction. The noise is assumed to be additive Gaussian with mean zero and variance of 0.3. The simulation scenario is shown in Fig. 5 .

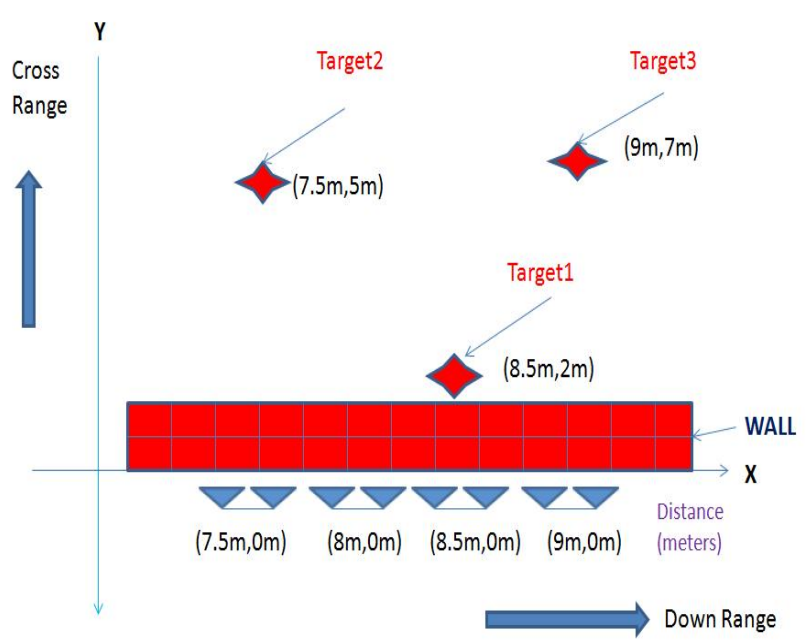

Fig. 5. Simulation Scenario 1

The wall is assumed to be concrete block and the two-way attenuation of the signal is considered to be $14 \mathrm{~dB}$. The targets are assumed to have reflectivity as $0.4,0.3$ and 0.5 respectively. All the antennas are assumed to get a reflection from the target. The antenna transmits frequencies in steps and received signal will be delayed depending on the distance from the antenna location to the target. The received signals are assumed to be processed by the receiver chain and the resulting base band signal containing phase values will be sampled by the ADC. The phase values corresponding to each step frequency will be collected for a given sensor. This process will be repeated for all the sensor positions. An IFFT is applied over these phase samples which indicate the distance to the target. These IFFT output values are passed through the back projection algorithm and the output is shown in Fig. 6. The back projection output indicates the presence of three targets with target 3 located at $(9,7) \mathrm{m}$ showing maximum intensity. We need to note the signal strength has decreased appreciably due to the wall effect. In real time scenario apart from the effect of wall many other factors such as selfcoupling and leakage effects need to be considered.

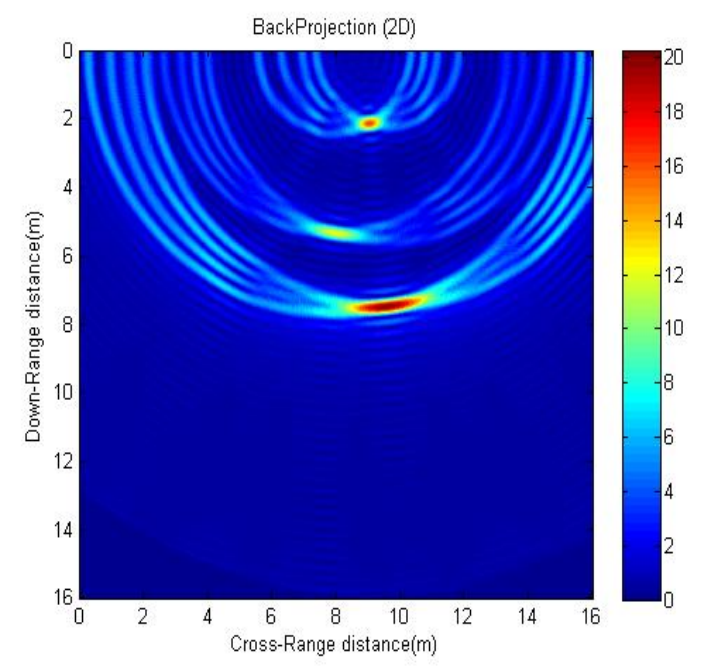

Fig. 6. Back Projection output of Scenario 1

\section{B. Scenario 2:}

In the second simulation scenario the aim is to detect the presence of human by means of heart rate and breathing detection. The respiration and heart beat rate have spectral content in the band of $[0.1,3] \mathrm{Hz}$. The presence of Doppler energy in this band indicates human identification. The simulation parameters are the same as that of the first scenario. The wall is assumed to provide a two-way attenuation of $14 \mathrm{~dB}$. The human target is at a distance of $3 \mathrm{~m}$ from the antenna array as shown in Fig. 7. The signal received from the target is modelled as described in section III.

$$
x(t)=m_{r} \sin \left(\omega_{\mathrm{r}} \mathrm{t}\right)+m_{h} \sin \left(\omega_{h} t\right)
$$

where $f_{r}=0.3 \mathrm{~Hz}$ corresponds to respiration rate and $f_{h}=2 \mathrm{~Hz}$ corresponds to heart beat of human. The data is collected for 256 sweeps with a sampling rate of $100 \mathrm{~Hz}$ where each sweep consists of 50 steps each. Hence the size of data array is 256X50. A 1024 point IFFT is applied over each sweep and the resulting matrix is of length $256 \times 1024$. 


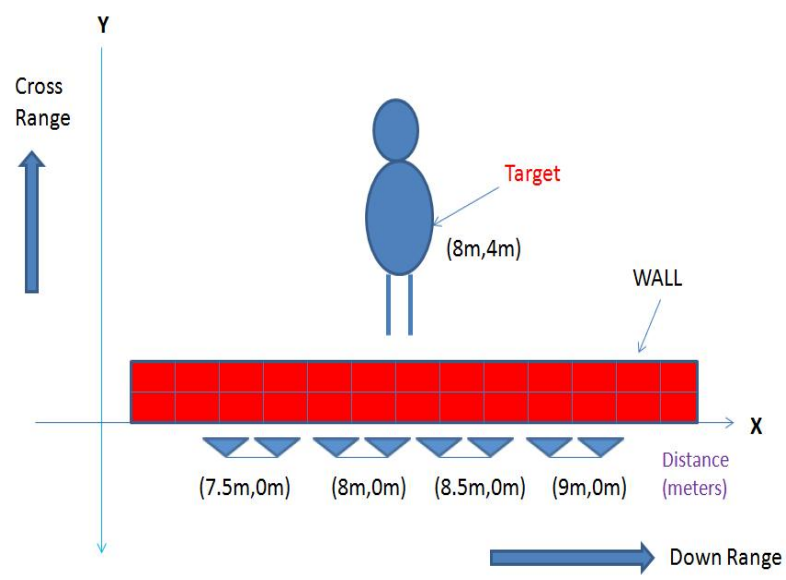

Fig.7. Simulation Scenario 2

In order to detect the micro Doppler, 256 point FFT was taken along the range bins through 256 sweeps. The RangeDoppler plane showing breathing rate of $0.5 \mathrm{~Hz}$ and $2 \mathrm{~Hz}$ of Heart beat is shown in the Fig. 8. All the values of the first row of range-Doppler plot, which corresponds to the zero ${ }^{\text {th }}$ filter, have been forced to zero in order to suppress the stationary clutter and enhance the other Doppler frequencies.

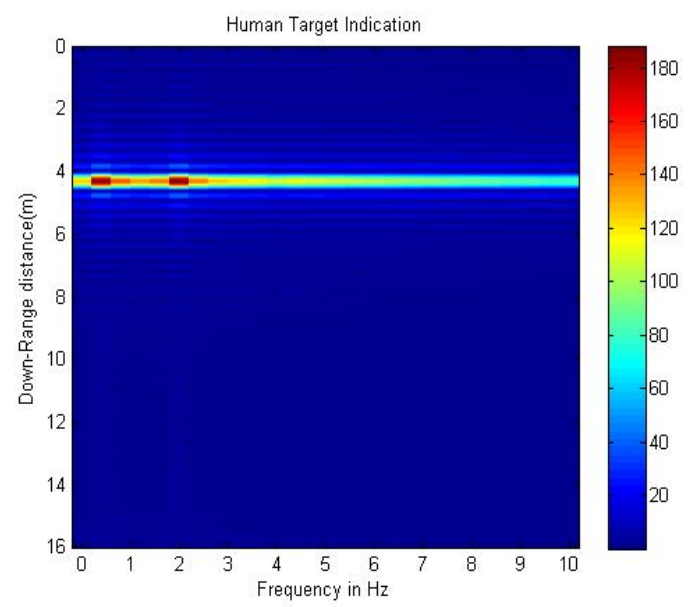

Fig.8. Human detection using Heart-beat and Respiration

As we can see from the Fig. 8, the heart beat has a Doppler frequency of $2 \mathrm{~Hz}$ and respiration rate has a Doppler frequency of $0.3 \mathrm{~Hz}$. This information can be used to indicate the presence of human at the down range of $4 \mathrm{~m}$.

\section{CONCLUSION}

In this paper, SFCW processing of targets has been discussed. Results of imaging through the walls by using well established Radar imaging techniques like Back projection is illustrated. Modeling of the Doppler echo due to the respiration and heart beat is proposed. The signal processing algorithm to extract the information is also discussed. As future work, these algorithms will be developed in real time using a Field Programmable Gate Array (FPGA) framework.

\section{ACKNOWLEDGMENT}

The authors express sincere acknowledgments to Dr.M.Z.I.SAJID for providing valuable guidance and necessary infrastructure.

\section{REFERENCES}

[1] Mones Amin, "Through-the-Wall Radar Imaging", CRC Press, 2010.

[2] A. R. Hunt, "Image formation through walls using a distributed radar sensor array," in Proc. 32nd IEEE AIPR Workshop, 2003, pp. 232237.

[3] G. L. Charvat, "A Low-Power Radar Imaging System," Ph.D. dissertation, Department of Electrical and Computer Engineering, Michigan State University, East Lansing, MI, August 2007.

[4] S. Gauthier and W. Chamma, "Through-The-Wall Surveillance," Technical Memorandum DRDC, Ottawa, Oct. 2002.

[5] Michael Aftanas, "Through wall imaging with UWB radar system", Ph.D. dissertation, Faculty of Electrical Engineering and Informatics Technical University of Kosice, Slovakia, August 2009.

[6] C. Li, Y. Xiao, J. Lin, "Experiment and spectral analysis of a lowpower Ka-band heartbeat detector measuring from four sides of a human body", IEEE Transactions on Microwave Theory and Techniques, vol.54, No.12, pp. 4464-4471, December 2006.

[7] J. Silvious J. Clark,T. Pizzillo,and D. Tahmoush, "Micro-Doppler phenomenology of humans at UHF and Ku-band for biometric characterization," Proc. of SPIE, vol. 7308, 2009.

[8] N. Maaref, P. Millot, C. Pichot, and O. Picon, "A study of UWB FM$C W$ radar for the detection of human beings in motion inside a building." IEEE Transactions on Geoscience and Remote Sensing, 47(5): 1297-1300, May 2009.

[9] J.Ferris, D.D and N.Currie, "A survey of current technologies for through-the-wall surveillance (TWS)",in Proc.SPIE 3577, Boston , MA, 1999,pp. 66-72.

[10] William H. Hayt and John A. Buck. Engineering electromagnetics. McGrawHill, 6th edition, December 2001. 